\title{
Alteration of Fecal Microbiota in Patients With Postinfectious Irritable Bowel Syndrome
} (Gut 2014;63:1737-1745)

\author{
Young-Eun Joo \\ Department of Internal Medicine, Chonnam National University Medical School, Gwangju, Korea
}

\section{Summary}

Irritable bowel syndrome (IBS) is a common, functional gastrointestinal disorder, which affects approximately 10-20\% of the general population. ${ }^{1-3}$ Despite its high prevalence, the pathogenesis of IBS is still very little understood, and seems to be multi-factorial disease. ${ }^{1-3}$ Many factors have been proposed to contribute to the pathogenesis of IBS, including visceral hypersensitivity, gastrointestinal dysmotility, activation of mucosal immune system, alterations of the gut microbiota, and impaired intestinal barrier function, as well as psychosocial distress. ${ }^{1-3}$ Recently, a newly identified cause for IBS is infectious gastroenteritis. ${ }^{1-3}$ About $10 \%$ of patients with IBS develop the symptoms consistent with diarrhea-predominant IBS (IBS-D) after an episode of infectious gastroenteritis and these patients are designated as postinfectious IBS (PI-IBS). ${ }^{4-6}$ Jalanka-Touvinene et $\mathrm{al}^{7}$ investigated the alteration of fecal microbiota composition and its association with clinical features in patients with PI-IBS. Compositional analysis of fecal microbiota showed the 27 discriminant bacterial groups, providing an index of microbial dysbiosis (IMD), which significantly separated the fecal microbial profile of PI-IBS patients from that of healthy control (HC), and the fecal microbiota of PI-IBS resembled that of IBS-D. The major differences in the fecal microbiota of PI-IBS compared to $\mathrm{HC}$ were the increase of the several members of Bacteroides and decrease of uncultured Clostridiales compared with HC. These descriminant microbial profiles, creating IMD positively correlated with intestinal symptoms, but not psychological symptoms. Also, IMD correlated with host mucosal gene expression including amino acid synthesis for gut integrity, cell junction pathways and inflammatory cytokine production, indicative of an increased inflammatory response and impaired intestinal barrier function in PI-IBS. The authors concluded that the fecal microbiota composition of patients with PI-IBS differs from that of $\mathrm{HC}$, and resembles that of IBS-D. Host-microbe associations between host gene expression and altered fecal microbiota suggest that impaired intestinal barrier function may underlie both immunological and microbiological deviations in the pathogenesis of PI-IBS.

\section{Comments}

IBS is a clinically heterogenous disorder, characterized and subclassified by symptoms including abdominal pain, bloating, constipation, diarrhea or alternating constipation and diarrhea. ${ }^{1-3}$

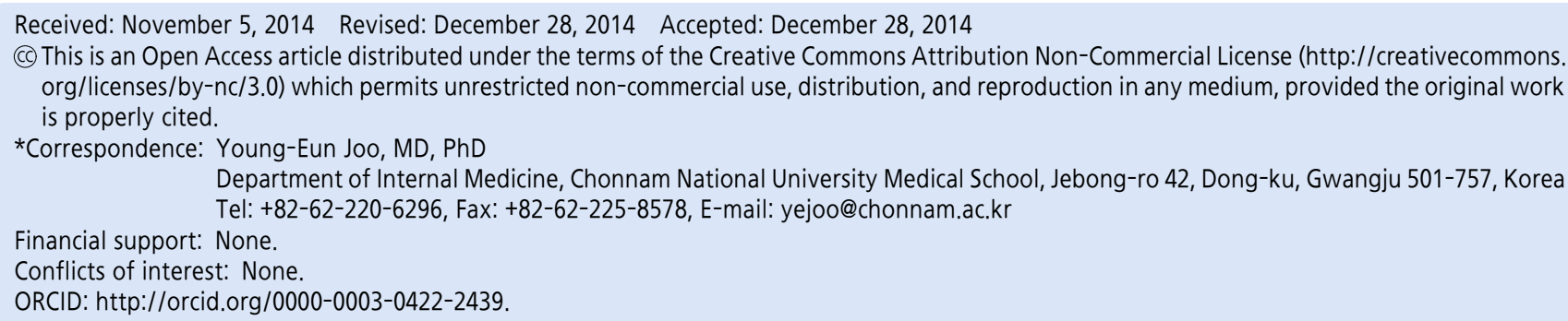


Until now, there has been increasing interest in trying to identify potential mechanisms in IBS because of its heterogeneity in symptoms and clinical phenotypes, and this endeavor has been driven by recent evidence that gut microbiota composition is altered in IBS patients compared to $\mathrm{HC}$ and this alteration influences the clinical phenotypes and host immune response in IBS patients. $^{8,9}$

PI-IBS is a functional gastrointestinal disorder which begins after an acute episode of infectious gastroenteritis. The vast majority of patients with infectious gastroenteritis recover completely within few days, but approximately $3.7-36.0 \%$ of patients experience persistent intestinal symptoms including abdominal pain and diarrhea which progress to PI-IBS. The most common infectious agents involved in the development of PI-IBS are Salmonella, Shigella, and Campylobacter, and the predominant bowel pattern noted is IBS-D. ${ }^{4-6}$

Jalanka-Touvinene et $\mathrm{al}^{7}$ identified the 27 discriminant bacterial genera, providing IMD, which significantly separated the fecal microbial profile of PI-IBS patients from that of $\mathrm{HC}$, and showed that the fecal microbial profile of patients with PI-IBS included the increase of Bacteroides and decrease of uncultured Clostridiales differing significantly from that of $\mathrm{HC}$, and resembled that of IBS-D. In this study, IMD correlated with the severity of intestinal symptoms including abdominal pain, bowel movement frequency, loose stools and multiple somatic symptoms except for psychological symptoms such as anxiety or depression, indicating that IMD may be a useful objective measure of disturbed bowel function in IBS. ${ }^{7}$ These results suggest that alteration of microbiota composition may play an important role in the pathogenesis of IBS in general, and PI-IBS in particular. Also, the difference of fecal microbial profiling such as IMD can allow the development of clinically useful subclassification of current IBS groups into patients with a predominant peripheral gut abnormality and patients with a predominant central nervous system basis for IBS in the future. ${ }^{7,10}$

The gut microbiota play an essential role to maintain normal gastrointestinal function and generate the intestinal immune response by promoting both immune activity and regulatory effects for protecting the host from pathogens. ${ }^{11,12}$ In this study, the host-microbe associations between host gene expression and altered fecal microbiota were investigated. The IBS-type microbiota positively correlated with increased inflammatory markers including eotaxin and mast cells, and increased expression of chemokine production and $\mathrm{B}$ cell antigen receptor signaling pathways, indicative of activation of mucosal immunity and inflamma- tion, provoked by altered microbiota in PI-IBS. ${ }^{7}$

Also, the IBS-type microbiota such as Bacteroides species negatively correlated with the glycine, serine and threonine metabolism pathways, important for maintaining the gut integrity and pathway regulating cell junctions, and positively correlated with a transcription factor tyrosine kinase oncogene, emerged as a key regulator of tight junction permeability. ${ }^{7}$ These findings indicate that both impaired intestinal barrier function and increased epithelial permeability are involved in the pathogenesis of PI-IBS. Consequently, PI-IBS may be based on luminal antigen penetration through an impaired intestinal barrier function leading to mucosal immune activation and inflammation.

However, this study showed that fecal microbiota alterations are important for only some subgroups of IBS including IBS-D, PI-non bowel dysfunction, PI-bowel dysfunction and PI-IBS. Therefore, further studies are needed to evaluate the fecal microbial profiling of other subgroups of IBS such as constipation-predominant IBS or mixed IBS with clinicopathophysiological features in large group of patients. ${ }^{10}$ In future, subgrouping of IBS based on the fecal microbiota alterations may be helpful to manage and treat the IBS patients.

In summary, altered composition of the microbiota, host immune activation and inflammation, increased epithelial permeability and impaired intestinal barrier function may contribute as the important pathogenic and pathophysiologic factors of PI-IBS. Fecal microbial profiling can allow the development of clinically useful subclassification of current IBS groups into patients with a predominant peripheral gut abnormality and patients with a predominant central nervous system basis for IBS. Therefore, modulation of gut microbiota may provide a potential therapeutic target for this condition in future.

\section{References}

1. Catanzaro R, Occhipinti S, Calabrese F, et al. Irritable bowel syndrome: new findings in pathophysiological and therapeutic field. Minerva Gastroenterol Dietol 2014;60:151-163.

2. Ringel $\mathrm{Y}$, Maharshak N. Intestinal microbiota and immune function in the pathogenesis of irritable bowel syndrome. Am J Physiol Gastrointest Liver Physiol 2013;305:G529-G541.

3. Han DS. Current status and prospects of intestinal microbiome studies. Intest Res 2014;12:178-183.

4. Grover M, Camilleri M, Smith K, Linden DR, Farrugia G. On the fiftieth anniversary. Postinfectious irritable bowel syndrome: mechanisms related to pathogens. Neurogastroenterol Motil 2014;26:156167.

5. Spiller R, Garsed K. Postinfectious irritable bowel syndrome. Gastroenterology 2009;136:1979-1988. 
6. Halvorson HA, Schlett CD, Riddle MS. Postinfectious irritable bowel syndrome - a meta-analysis. Am J Gastroenterol 2006;101: 1894-1899.

7. Jalanka-Tuovinen J, Salojärvi J, Salonen A, et al. Faecal microbiota composition and host-microbe cross-talk following gastroenteritis and in postinfectious irritable bowel syndrome. Gut 2014;63:17371745.

8. Jeffery IB, O'Toole PW, Öhman L, et al. An irritable bowel syndrome subtype defined by species-specific alterations in faecal microbiota. Gut 2012;61:997-1006.

9. Rajilić-Stojanović M, Biagi E, et al. Global and deep molecular anal- ysis of microbiota signatures in fecal samples from patients with irritable bowel syndrome. Gastroenterology 2011;141:1792-1801.

10. Simrén M. IBS with intestinal microbial dysbiosis: a new and clinically relevant subgroup? Gut 2014;63:1685-1686.

11. Collins SM. A role for the gut microbiota in IBS. Nat Rev Gastroenterol Hepatol 2014;11:497-505.

12. Bonfrate L, Tack J, Grattagliano I, Cuomo R, Portincasa P. Microbiota in health and irritable bowel syndrome: current knowledge, perspectives and therapeutic options. Scand J Gastroenterol 2013;48:995-1009. 